Martin Hellwig*

\title{
Deutschland braucht ein Investitionskompetenzprogramm
}

Die Autobahnbrücke über den Rhein bei Leverkusen ist seit 2014 für Fahrzeuge mit mehr als 3,5 t Gesamtgewicht gesperrt. Der Auftrag für den Neubau wurde dieser Tage neu vergeben. Man hofft, dass die neue Brücke damit bis Ende 2023 fertig gestellt werden kann. Dann sind es neun Jahre Sperrung für Lkws. Die durch die Sperrung bedingten Umwege sind mit großen Kosten verbunden, für die Unternehmen, die Anwohner:innen und die Umwelt. Die Brücke ist von zentraler Bedeutung für Transporte aus Belgien, Nordfrankreich und England. Als sie 1965 errichtet wurde, rechnete man mit 40.000 Kraftfahrzeugen pro Tag, daraus wurden 120.000 pro Tag, davon 14.000 Lkws. Man hat es versäumt, rechtzeitig über einen Ersatz der Brücke nachzudenken. Dass die Brücke viel mehr aushielt als ursprünglich gedacht, auch viel länger, wurde dankbar wahrgenommen, dass das nicht ewig so gehen würde, wurde verdrängt. Eine 2012/2013 durchgeführte Sanierung war nicht nachhaltig. Der Planfeststellungsbeschluss für den Neubau erfolgte 2016, die Bestätigung durch das Bundesverwaltungsgericht 2017. Der erste Vertrag wurde 2020 wegen Mängeln an importierten Stahlbauteilen gekündigt; jetzt hofft man, dass der neue Vertrag problemlos umgesetzt wird.

(C) Der/die Autor:in(nen) 2021. Open Access: Dieser Artikel wird unter der Creative Commons Namensnennung 4.0 International Lizenz veröffentlicht (creativecommons.org/licenses/by/4.0/deed.de).

Open Access wird durch die ZBW - Leibniz-Informationszentrum Wirtschaft gefördert.

* Der Artikel stützt sich maßgeblich auf das im Juni 2020 verabschiedete Gutachten „Öffentliche Infrastruktur in Deutschland: Probleme und Reformbedarf" des Wissenschaftlichen Beirats beim Bundesministerium für Wirtschaft und Energie, an dessen Erstellung der Verfasser mitgewirkt hat. Die Belege für viele Aussagen finden sich in diesem Gutachten. Zum Thema Schuldenbremse und öffentliche Investitionen ist die hier geäußerte Meinung nicht dem Beirat zuzuschreiben; das Beiratsgutachten dokumentiert dazu einen Dissens.

Prof. Dr. Martin Hellwig ist emeritierter Direktor am Max-Planck-Institut zur Erforschung von Gemeinschaftsgütern in Bonn. Er war Vorsitzender des Beratenden Wissenschaftlichen Ausschusses beim Europäischen Ausschuss für Systemrisiken (European Systemic Risk Board - ESRB).
Verschieben von Ersatzinvestitionen, Langsamkeit der Planung, Langsamkeit des Rechtswegs, Probleme bei der Vertragsgestaltung und -umsetzung - da denkt man nicht nur an die Leverkusener Brücke, sondern auch an die Elbphilharmonie und den Flughafen Berlin Brandenburg (BER). Deutschland hat nicht nur eine Investitionslücke, sondern auch eine Investitionskompetenzlücke. Investitionslücken und Investitionskompetenzlücken treten nicht nur bei Großprojekten auf. Diese fallen besonders auf, aber die Masse der Probleme dürfte im Kleinen liegen, bei den Kommunen, die immer noch für die Hälfte der staatlichen Investitionen in Deutschland aufkommen.

\section{Defizite}

Der Anteil der staatlichen Bruttoanlageinvestitionen (ohne FuE) am Bruttoinlandsprodukt (BIP) in Deutschland ist zwischen 1992 und 2018 von 2,9\% auf 1,7\% gesunken. 1970 hatte dieser Anteil noch bei 4,7\% gelegen, 1980 bei 3,5\%, 1989 bei 2,5 \%. ${ }^{1}$ Die absoluten Werte dieser Bruttoanlageinvestitionen stiegen in diesem Zeitraum um $28 \%$, von 46,3 Mrd. Euro auf 59,4 Mrd. Euro - bei gleichzeitigem Wachstum der Preise für Bauleistungen um $62 \%$, der Erzeugerpreise gewerblicher Produkte um $33 \%$.

Der Rückgang fand vor allem in den Kommunen statt. Deren Investitionen lagen 2018 nominal (!) auf ähnlicher Höhe wie 1992, bei ca. 28 Mrd. Euro. Zwischenzeitlich, von 2000 bis 2017, lagen sie jeweils noch um mehrere Milliarden niedriger. Nach den im KfW-Kommunalpanel ermittelten Einschätzungen ist der Investitionsrückstand der Kommunen von ca. 80 Mrd. Euro im Jahr 2009 auf 147 Mrd. Euro im Jahr 2019 angestiegen. Hinter diesen Zahlen verbergen sich unter anderem schlecht reparierte Straßen, undichte Schuldächer und marode Abwasserkanäle.

Die Kommunen haben auch die für die Planung und Durchführung von Investitionsprojekten erforderlichen Personalkapazitäten reduziert; z. B. in den Bauämtern von 1995 bis 2015 um ca. $40 \%$ der Vollzeitäquivalente. Daher sind heute viele Kommunen gar nicht mehr in der Lage, größere Bauinvestitionen angemessen zu planen und durchzuführen. Deshalb waren aus dem 2015 errichteten Kommunalinvestitionsförderungsfonds des Bundes

1 Den älteren Zahlen lag eine engere statistische Definition zugrunde; der tatsächliche Rückgang war daher wohl noch größer, als die Zahlen inn erscheinen lassen. Der Anstieg von 1989 auf 1992 spiegelt den Aufbau Ost nach der Vereinigung. 
bis Anfang 2019 nicht einmal die Hälfte der verfügbaren Mittel abgeflossen.

Dabei gibt es große Unterschiede zwischen den Kommunen, auch zwischen den Ländern. Im Querschnittsvergleich sind vor allem die Kommunen besonders betroffen, die Finanzprobleme haben. In den alten wie den neuen Bundesländern betrifft das insbesondere Kommunen und Regionen, deren Wirtschaft früher florierte, die aber heute eine relativ hohe Arbeitslosigkeit aufweisen, wo die Kommunen hohe, vom Bund festgelegte Sozialleistungen zu erbringen haben. Diese Kommunen scheinen sich mit Krediten und mit einer Rücknahme der Investitionen durchzulavieren.

Auch die zur Qualität der Infrastruktur zur Verfügung stehenden Daten geben zu Pessimismus Anlass - obwohl die finanzielle Entwicklung hier deutlich günstiger war. In den Verkehrsinvestitionsberichten des Bundesministeriums für Verkehr und digitale Infrastruktur werden die Substanzwerte von ca. $17 \%$ der Bundesautobahnen und ca. $30 \%$ der Bundesfernstraßen als „schlecht“ oder „sehr schlecht" bezeichnet. Die Gebrauchswerte sind deutlich besser, das lässt vermuten, dass viele Reparaturen nur die Glätte der Straßenoberfläche wiederherstellen, ohne zugrundeliegende bauliche Schäden zu beheben. Es ist dann nur eine Frage der Zeit, bis teure Grundsanierungen fällig werden. Der Bauwerkszustand der Brücken wird zu $12 \%$ als „nicht ausreichend“ oder „ungenügend“ bezeichnet, zu $33 \%$ als „ausreichend“.2

Die Inzidenz von Staus, gemessen in Staukilometern, hat sich von 2002 bis 2018 fast verfünffacht. Dementsprechend gaben 2018 in einer Unternehmensbefragung des Instituts der Deutschen Wirtschaft $72 \%$ der Befragten an, die Geschäftsabläufe würden durch Infrastrukturmängel im StraBenverkehr beeinträchtigt; 2013 waren es noch $64 \%$.

Bei Wasserstraßen und Schienenverkehr sieht es ähnlich aus. Das Durchschnittsalter von Schleusen und Wehren liegt bei über 65 Jahren, die alten Anlagen fallen immer häufiger aus. Das Durchschnittsalter der Brücken im Schienenverkehr wurde bis 2016 mit 56 Jahren ausgewiesen, nach einer Datenkorrektur 2017 mit über 73 Jahren. Die von der Deutschen Bahn (DB AG) ausgewiesenen Verspätungsminuten sind von 2009 bis 2018 von 125 Minuten pro 1.000 gefahrene Kilometer auf 184 Minuten angestiegen.

2 Für den kommunalen Bereich bezeichnete das Deutsche Institut für Urbanistik 2013 ca. 10.000 von 67.000 Brücken als nicht mehr sanierungsfähig; etwa $19 \%$ fielen in die Kategorien „nicht ausreichend“ oder „ungenügend“.
Die DB AG selbst weist regelmäßig Verbesserungen bei den Qualitätskennzahlen „Theoretischer Fahrzeitverlust“ und „Anzahl Infrastrukturmängel“ aus. Nach Einschätzung des Bundesrechnungshofs sind diese Qualitätskennzahlen allerdings wenig aussagekräftig, intransparent und manipulierbar und spiegeln weniger die tatsächliche Qualität der Infrastruktur als vielmehr die Interessen der DB AG bei der Umsetzung der sogenannten Leistungs- und Finanzierungsvereinbarungen zur Regelung der Zuschüsse des Bundes zur Finanzierung von Netzinvestitionen an die DB AG wider. Nach diesen Vereinbarungen werden keine Einzelnachweise über die Mittelverwendung erbracht, sondern nur die Einhaltung der Qualitätskennzahlen überprüft.

Von Zukunftsinvestitionen ist bei alledem noch gar nicht die Rede, weder für Strom- und Gasnetze im Zuge der Energiewende noch für die Telekommunikationsinfrastruktur als Grundlage der Digitalisierung noch für die Gesundheitsinfrastruktur nach Corona. Bei den Energieund Telekommunikationsnetzen wird sich ähnlich wie bei der DB AG die Frage stellen, wie öffentliche Interessen wahrzunehmen sind, wenn sie nicht mit den Privatinteressen der Unternehmen zusammenfallen.

\section{Gründe für Fehlentwicklungen}

Die Rückgänge der 1980er Jahre und des Jahrzehnts von 1995 bis 2005 spiegelten nicht einen Rückgang des Bedarfs, sondern die Notwendigkeit von Haushaltskonsolidierungen wider. Die Expansion der öffentlichen Haushalte in den 1960er und 1970er Jahren hatte die Sozialausgaben deutlich ansteigen lassen, die nachfolgende Konsolidierung ging vor allem zulasten der Investitionen, dies übrigens auch in anderen OECD-Ländern. Im Fall der Kommunen wirkte sich auch die Belastung durch die Steuerreform von 2000 aus.

In der Praxis sind Investitionen eine Residualgröße der Haushaltsplanung. Man fährt sie je nach Kassenlage hinauf oder hinunter. Auch das Wachstum nach 2015 dürfte vor allem mit der Konjunktur zu tun haben und in einer Konsolidierung nach Corona wieder zurückgefahren werden. Eine solche Stop-and-Go-Strategie ist ineffizient. Zum einen sind Planungskapazitäten, die eingespart wurden, nicht ohne weiteres wiederaufzubauen. Die Reduktion der Planungskapazitäten in den Jahren vor 2005 dürfte erheblich zu späteren Planungsfehlern, Kostenüberschreitungen und Ausführungsverzögerungen beigetragen haben. Zum anderen ist zu erwarten, dass die Ausgabenerhöhungen bei guter Kassenlage vor allem die Preise ansteigen lassen, denn die Anbieter:innen haben beschränkte Kapazitäten, und bei guter Konjunktur steht die Nachfrage des Staats in Konkurrenz zur privaten Nachfrage. Ein 
Teil des Ausgabenwachstums seit 2015 dürfte in einem Anstieg der Preise für Bauleistungen verpufft sein.

Anders als private Güter werden öffentliche Leistungen zumeist nicht durch Zahlungen der Nutzer:innen finanziert. Der Bedarf ergibt sich daher nicht aus der Zahlungsbereitschaft der Kunden. Der politische Diskurs tritt an die Stelle des Marktes. Das bringt verschiedene Probleme mit sich:

- Da Nutzung und Finanzierung auseinanderfallen, gibt es immer Verteilungskonflikte. Die einen profitieren mehr als sie beitragen, die anderen weniger. Dritte schließlich nutzen die Gelegenheit zur politischen Profilierung, da sie grundsätzlich andere Vorstellungen haben.

- Öffentliche Debatten befassen sich bevorzugt mit Steuer- und Sozialpolitik. "Öffentliche Investitionen“ dagegen sind entweder ein abstrakter Sammelbegriff, unter dem man sich nichts vorstellen kann, oder so speziell, dass nur wenige sich betroffen fühlen.

- Bei steuerfinanzierten langlebigen Investitionen ist ein Teil der Nutzer:innen an den Entscheidungen nicht beteiligt, da diese Personen erst in der Zukunft am politischen Prozess teilnehmen, anders als die heutigen Steuerzahler:innen und Wähler:innen.

- Bis Einsparungen bei Investitionen spürbare Folgen für die Leistungsfähigkeit der Infrastruktur haben, vergeht gewöhnlich einige Zeit. Für den Politiker, der bald zur Wiederwahl ansteht, besteht die Versuchung, lieber bei Investitionen zu sparen als bei Ausgaben, deren Kürzungen unmittelbar wahrgenommen werden. Ausgenommen sind spektakuläre, medial wirksame Großprojekte, die dann den Vorzug bekommen gegenüber langweiligen Erhaltungs- und Ersatzinvestitionen.

- Politik und Verwaltung schwanken zwischen Allmachtvorstellungen bei der Formulierung von Gestaltungswünschen mit etlichen oft teuren Änderungen (Elbphilharmonie, BER) und der Scheu vor Verantwortung, etwa bei der Kontrolle der Mittelverwendung durch die DB AG.

- Die Verrechtlichung der Entscheidungsprozesse und die Dauer des Rechtswegs erhöht die Veto-Macht der Gegner und die Komplexität und die Kosten der Planung. Zu nennen sind nicht nur nachbarschaftsrechtliche oder umweltrechtliche, sondern auch wettbewerbs- und ausschreibungsrechtliche Vorschriften, die eine Aufteilung in kleine Losgrößen erzwingen mit Kosten für die Koordination der Auftragnehmer oder eine Fokussierung auf den Preis zulasten der Qualität.

\section{Reformbedarf}

Die angesprochenen Probleme betreffen teilweise die föderale Ordnung der Bundesrepublik. Es ist ein Unding, dass Bund und Länder den Kommunen Aufgaben zuweisen, ohne die Kosten dafür zu übernehmen. Hier muss Abhilfe geschaffen werden.

Für das zentrale Desiderat einer Förderung und Verstetigung der öffentlichen Investitionen dürfte das nicht ausreichen. Der Wissenschaftliche Beirat beim Bundesministerium für Wirtschaft und Energie hat daher die Schaffung von Investitionsfördergesellschaften (IFG) vorgeschlagen, Institutionen des Bundes und/oder der Länder. Diese sollten jeweils vom zuständigen Gesetzgeber bindende vertragliche oder gesetzliche Ansprüche auf gleichbleibende Mittelzuweisungen über einen längeren Zeitraum bekommen und diese Mittel den Kommunen, auf Antrag für Investitionsprojekte zur Verfügung stellen. Die Verstetigung der Mittelzuweisungen durch die IFG würde den Kommunen und den Anbietern der benötigten Leistungen, z.B. der Bauwirtschaft, Planungssicherheit geben. Die Wirkungen des Jährlichkeitsprinzips der staatlichen Haushalte würden deutlich gemildert. IFG könnten auch das Problem der Knappheit an qualifiziertem Personal bei den Kommunen mildern. Die gemeinsame Nutzung von Personal der IFG durch die Kommunen eines oder mehrerer Bundesländer dürfte Größen- und Verbundvorteile erzeugen, denn nicht jede Kommune ist groß genug und nimmt so regelmäßig größere Investitionen vor, dass ein Vorhalten des erforderlichen Personals sinnvoll ist.

Ein zentrales Problem betrifft die Governance solcher Gesellschaften. Auf der einen Seite muss die Initiative bei den Antragstellern liegen, desgleichen die Letztkompetenz zur Entscheidung über die Ausgestaltung der jeweiligen Projekte. Auf der anderen Seite sollte die IFG in der Lage sein, über die Konditionalität der Mittelvergabe Missbräuche bei der Planung, Auftragsvergabe und Durchführung zu verhindern. Die Leitungs- und Kontrollgremien müssen die Gewähr dafür bieten, dass beide Anliegen vernünftig austariert werden.

Ähnliches gilt für die Governance der Netze. Bei der DB AG sollte die Governance sicherstellen, dass die öffentlichen Belange, die die Finanzierung des Netzes durch den Bund begründen, auch tatsächlich durchgesetzt werden. Bei den anderen Netzen ist es nötig, die öffentlichen Belange mit angemessener Finanzierung und Durchsetzung einzubringen. Es gilt, die institutionellen Rahmenbedingungen so anzulegen, dass die politisch Verantwortlichen die Projekte gestalten können, dies aber mit genügend Transparenz und Verbindlichkeit, sodass die Wirkungen der Launenhaftigkeit der politischen Diskurse eingeschränkt werden. 


\section{Schuldenbremse und öffentliche Investitionen}

Früher war es zulässig, öffentliche Investitionen durch Kredite zu finanzieren. Die 2009 ins Grundgesetz eingeführte Schuldenbremse hat diese Möglichkeit beseitigt. 2020 wurde die Schuldenbremse wegen Corona ausgesetzt. Jetzt läuft eine Diskussion darüber, ob man sie nicht überhaupt abschaffen sollte.

Das schon vor 2009 geltende allgemeine Kreditaufnahmeverbot sollte die Politik daran hindern, willkürlich Schulden zu machen auf Kosten der nächsten Generation. Man kann sich aber auch zulasten der nächsten Generation bereichern, indem man die Infrastruktur verrotten lässt. Die bis 2009 geltende Ausnahme für Investitionen sollte das verhindern. Ohne dieses Korrektiv ist die politische Willensbildung zulasten der Investitionen verzerrt.

Die Ausnahme wurde abgeschafft, weil sie vielfach missbraucht wurde, unter anderem durch eine gewisse Willkür bei der Definition dessen, was als Investition behandelt wurde. Es ist allerdings nicht einzusehen, warum solche Missbräuche eine Änderung der Grundsatzregelung begründen und nicht durch eine Reform der Governance behoben werden können. Ein Beispiel wäre eine Prozedur der Zertifizierung des Investitionscharakters bestimmter Ausgaben durch eine unabhängige Institution, etwa die IFG. 